\title{
Alcohol Consumption: A New Risk Factor for Arterial Stiffness?
}

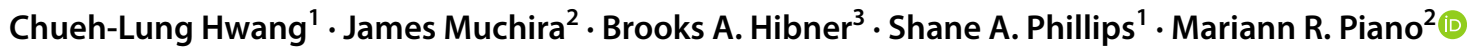

Received: 17 December 2021 / Accepted: 4 February 2022 / Published online: 23 February 2022

(c) The Author(s), under exclusive licence to Springer Science+Business Media, LLC, part of Springer Nature 2022

\begin{abstract}
The relationship between alcohol consumption and cardiovascular disease risk is complex. Low-to-moderate daily alcohol consumption (1-2 drinks/day) is associated with reduced risk, whereas greater amounts of alcohol consumption and a "binge" pattern of drinking are associated with increased cardiovascular risk and mortality. Arterial stiffness may help explain the complex relationship. This integrated review summarizes data from studies examining the associations between alcohol consumption and pulse wave velocity, a gold standard measure of arterial stiffness. We also briefly review the concept and methodology of pulse wave velocity measurement as well as the mechanisms of alcohol-induced arterial stiffening. Findings among the different studies reviewed were inconsistent with methodological challenges related to alcohol use assessment. While making specific conclusions regarding this relationship is tenuous; the data suggest that excessive alcohol consumption or a binge drinking pattern is associated with increased arterial stiffness.
\end{abstract}

Keywords Alcohol consumption $\cdot$ Arterial stiffness $\cdot$ Pulse wave velocity

\section{Introduction}

Arterial stiffness refers to arterial wall changes which arise from gradual fragmentation and loss of elastin fibers and accumulation of stiffer collagen fibers in the arterial wall causing functional impairment of the artery [1]. Data from numerous studies have demonstrated the prognostic importance of increased arterial stiffness and its mechanistic relationship to the development of vascular-related conditions such as hypertension and coronary artery disease [1]. A $1.0 \mathrm{~m} / \mathrm{s}$ increase in pulse wave velocity (PWV), a measure of arterial stiffness, is associated with an increased risk of total cardiovascular (CV) events (12\%-14\%), CV-related mortality $(13 \%-15 \%)$, and all-cause mortality $(6 \%-15 \%)[2,3]$. Arterial stiffness can serve as a powerful prognostic tool to

Handling Editor: Y. James Kang.

Mariann R. Piano

mariann.r.piano@vanderbilt.edu

1 Department of Physical Therapy, University of Illinois at Chicago, Chicago, USA

2 School of Nursing, Vanderbilt University, 46121 st Avenue South, 415 Godchaux Hall, Nashville, TN 37240-1119, USA

3 Department of Kinesiology and Nutrition, University of Illinois at Chicago, Chicago, USA help detect and manage CV disease since it (1) can be detectable before the onset of clinically apparent CV disease, (2) can be prevented, and (3) in some instances, reduced [2,3].

There are complex associations between alcohol consumption and the overall occurrence of certain CV conditions, which are strongly modulated by the dose and pattern of alcohol consumption. For instance, low-to-moderate daily alcohol consumption (i.e., $<15-20 \mathrm{~g} /$ day, $1-2$ standard drinks) is associated with a reduced risk of CV disease and mortality. In contrast, greater amounts of alcohol consumption and a "binge" pattern of drinking are associated with an increase in CV risk and mortality [4], resulting in a J-shaped relationship between alcohol use and CV disease. According to the 2019 National Survey on Drug Use and Health, 85.6\% Americans ( $\geq 18$ years) consume alcohol [5]. Twenty-five percent report binge drinking in the last month (defined as consuming 5 or more alcoholic drinks for males or 4 or more alcoholic drinks for females on the same occasion), and 15 million Americans had an alcohol use disorder [5]. Since the 2020 outbreak of the COVID-19 pandemic, there have been numerous reports of increased alcohol use and engagement in harmful patterns of drinking, such as binge drinking [6, 7]. These statistics and the clinical significance of arterial stiffness highlight the need to understand the association between alcohol consumption and arterial stiffness. 
This integrated review summarizes data from studies examining the associations between alcohol consumption and arterial stiffness. We also briefly review the concept and measurement of arterial stiffness. This review will fill a gap in our understanding of the potential association between alcohol consumption and arterial stiffness: a marker of premature vascular disease that may be altered in otherwise healthy populations who drink.

\section{Definition of Arterial Stiffness}

The term, arterial stiffness, refers to the elastic properties of arterial walls and is defined as the change in pressure for a given volume [3, 8]. It is the inversion of arterial compliance or distensibility and is complex, given the heterogenous wall properties within the arterial system. The central (elastic) arteries, large diameter arteries close to the heart, are very distensible due to their high elastin content. These elastic arteries buffer pulsatile energy from the forward pulse wave generated by each heart contraction, allow blood flow during diastole, and prevent end-organ damage [2,9]. Peripheral muscular (conduit) arteries are smaller in diameter and contain a large amount of smooth muscle and collagen (inelastic), while the smallest arteries (arterioles) are comprised chiefly of smooth muscle [2,9]. Therefore, arterial stiffness increases from the central arteries to arterioles [1], leading to impedance mismatch and reflection of forward energy (backwards) toward the heart. Consequently, the reflected energy impacts the aortic pressure waveform, ultimately affecting blood pressure and flow $[1,8]$.

\section{Physiological Mechanisms of Arterial Stiffening}

Arterial stiffening occurs due to structural changes throughout segments of the arterial tree. Arterial wall elasticity is dependent on the composition of elastin and collagen fibers. During periods of low pressure, arterial compliance relies on elastin, while more rigid collagen fibers are progressively recruited during increased pressure or stretch [1]. Arterial wall elastin is only formed during fetal growth and infancy with a half-life of 40-50 years [10-12]. During continuous higher-pressure states or simply with time (i.e., aging), elastin degrades $[1,2,12]$. Elastin degradation shifts the load to stiffer arterial wall components, collagen fibers [1, 12], stiffening the large elastic arteries [12]. In addition to these structural changes, smooth muscle cell stiffness, arterial calcification, inflammation, increased sympathetic nervous system activity, and endothelial dysfunction in the arteries contribute to functional arterial stiffening [1, 12]. Several factors and conditions associated with arterial stiffness are noted in Table 1.

\section{Methods of Assessing Arterial Stiffness}

Arterial stiffness is commonly assessed by PWV, a measure of how fast the pulse wave travels in a defined arterial segment. Several methodologies, including tonometry-based devices, oscillometry- or cuff-based devices, ultrasonography, and magnetic resonance imaging, exist to determine PWV. Briefly, PWV is determined by measuring either pressure or flow waveforms using a probe, tonometer, or cuff at different sites of arteries simultaneously or with electrocardiogram gating as reference [1], and then calculating the ratio of the distance between the recording sites to the time delay between the pulse waves. The measurement should be performed in duplicate by trained personnel after subjects' rest in a supine position for at least $10 \mathrm{~min}$. Carotid-tofemoral PWV (cfPWV) is the gold or reference standard of assessing arterial stiffness [13, 14] (Fig. 1). This is because there is significant evidence demonstrating an association with mortality and CV disease, independent of traditional risk factors [15-24]. A cut-off of $10 \mathrm{~m} / \mathrm{s}$ has been used to predict CV events [25]. However, instead of a single fixed number, recent evidence recommends the reference values of cfPWV specific to age and blood pressure groups, given that arterial stiffness is predominantly influenced by age and blood pressure [1, 26]. During normal aging, cfPWV increases non-linearly with a greater change after the age of 50 years [27], while at any age cfPWV is linearly related to blood pressure [26].

Although PWV can be measured in other arterial segments, such as carotid-radial or estimated from a single point measure, these measures are not recommended as they

Table 1 Factors and conditions known to be associated with arterial stiffening

Factors and conditions

Advancing age

Arterial hypertension

Metabolic diseases

Diabetes mellitus

Obesity

Metabolic associated fatty liver disease

Calcium and phosphate metabolism disorders

Connective tissue diseases

Oxidative stress

Life style factors

$\uparrow$ Salt intake

$\downarrow$ Physical activity 


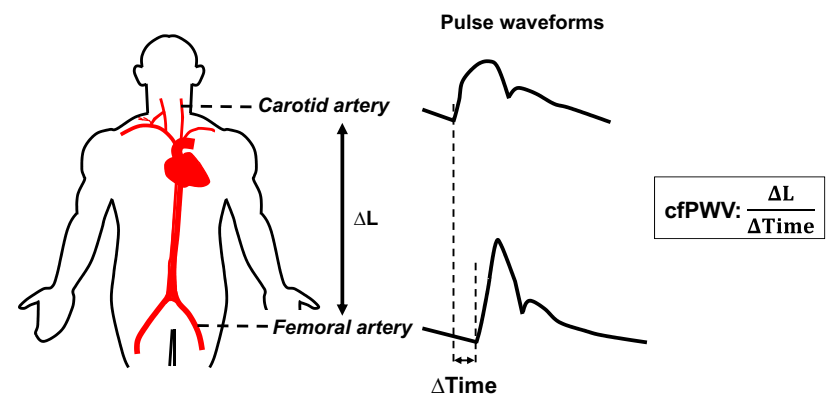

Fig. 1 The Measurement of carotid-to-femoral pulse wave velocity (cfPWV) as a non-invasive gold standard of arterial stiffness. Pressure or flow waveforms can be measured by using a probe, tonometer, or cuff at the carotid and femoral arteries simultaneously. cfPWV is calculated as the ratio of the distance between the recording sites to the time delay between the pulse waves. Adapted from [73]

have not been predictive of CV outcomes [1, 28]. In Asian populations, brachial-ankle PWV (baPWV) and its derived cardio-ankle vascular stiffness index are predictive of $\mathrm{CV}$ outcomes, although less is known in other populations [1, 28]. Augmentation index, or the ratio of the late systolic increase in the aortic pressure wave over pulse pressure, is sometimes recognized as a surrogate measure of arterial stiffness [8]. However, augmentation index is determined by arterial stiffness and other factors, especially heart rate [8]. In certain pathophysiological conditions augmentation index can change without a change in aortic PWV, therefore; augmentation index is not an accurate surrogate of arterial stiffness $[8,29]$.

\section{Data Sources and Search Strategy}

This integrative review mainly summarizes data from nonrandomized research studies designed to examine the association between arterial stiffness and alcohol consumption in adults ( $>15$ years of age). Specifically, we review data from human cross-sectional, case-control, and longitudinal epidemiological studies that have examined the association between arterial stiffness and alcohol consumption. We used the electronic databases PubMed/Medline and Google Scholar using a combination of the following main keywords: arterial stiffness, vascular aging, pulse wave velocity, alcohol consumption, vascular structure, vascular age, subclinical arterial damage, and CV disease. Studies were included in this integrated review if: (1) arterial stiffness was determined by measuring cfPWV or baPWV; (2) use of aforementioned study design; (3) alcohol consumption was quantified in drinks or grams/units of ethanol per day or week or month and; (4) reference or detailed methods for the measurement of arterial stiffness.

\section{Effects of Alcohol Consumption on Arterial Stiffness}

\section{Acute Alcohol Consumption Clinical Studies}

Two studies were identified that examined the 'acute' onetime effect of ingesting alcoholic beverages on arterial stiffness. Nishiwaki and colleagues randomly assigned young males $(N=11,20-22$ years) into different groups (over 4-days) and compared the effects of $200 \mathrm{ml}$ and $350 \mathrm{ml}$ of beer (alcohol content not indicated) vs. similar volumes of alcohol-free beer. No changes in cfPWV or baPWV were found after alcohol-free beer. However, cfPWV was significantly reduced following ingestion of both volumes of alcohol-containing beer to a similar level $(-0.6 \pm 0.2 \mathrm{~m} / \mathrm{s})$ (breath alcohol levels $\sim 13$ to $40 \mathrm{mg} \%$ ). The reduction in cfPWV was sustained 60 min following beer ingestion. A similar decrease was found in baPWV after ingestion of alcohol-containing beer [30]. In a double-blind randomized cross-over design, Mahmud and Feely examined cfPWV after the consumption of $500 \mathrm{ml}$ of red wine $(0.8 \mathrm{~g} / \mathrm{kg}$ ethanol, $\sim 3$ glasses of wine) in healthy men $(n=3)$ and women $(n=5)$ (aged 21-40 years) [31]. Compared to the ingestion of non-alcoholized wine, there was a significant decrease in cfPWV (from 7.6 to $6.9 \mathrm{~m} / \mathrm{s}$ ) $90 \mathrm{~min}$ after the ingestion of wine [31]. Collectively these findings indicate that a onetime ingestion of both small ( $\sim 1$ bottle of beer) and moderate ( $\sim 3$ glasses of wine) amounts of alcohol are associated with decreases in arterial stiffness.

\section{Cross-Sectional Studies}

Several population-based and clinical cross-sectional studies have examined the association between alcohol consumption and arterial stiffness with equivocal findings. Findings include: (1) no association of arterial stiffness with different alcohol consumption amounts [32-35], (2) an inverse or J-shaped association [36-39], and (3) significant increases associated with any amount of alcohol use [40] or a binge pattern of drinking [38, 41, 42].

In the Rotterdam Study of 3178 participants (57\% women; mean age 72 years), Mattace-Raso et al. did not find the association of alcohol consumption with cfPWV in men and women, after controlling for several known CV factors [35]. Basdeki et al. also found no association among different levels of alcohol intake ( $>0-10 \mathrm{~g} /$ day to $>30 \mathrm{~g} /$ day) among men $(n=408)$ and women $(n=530)$ (mean age across alcohol drinking groups 49-54 years) [32]. Among young adults (mean age 21 years, $N=50,70 \%$ women), Tisdel et al. reported an average of $14 \mathrm{~g} /$ day of alcohol (1 drink) was not associated with changes in cfPWV. However, energy from alcohol (percentage of total calories) was 
a significant predictor of increases in cfPWV [34]. Yu and colleagues assessed alcohol using a standardized questionnaire and categorized young adult (mean age 25 years) men $(n=79)$ and females $(n=74)$ into the following 4 groups: non-drinkers ( $<2$ drinks/week), light drinkers (2-6 drinks/ week), moderate drinkers (7-9 drinks/week for women, and 7-14 drinks/week for men), and heavy drinkers ( $>9$ drinks/ week for women, and $>14$ drinks per week for men) [33]. No differences in cfPWV were found among the alcohol groups [33].

Several studies report a J-shaped relationship between alcohol consumption and arterial stiffness. In two related studies, Sierksma and colleagues found that compared to abstainers, 10-14 drinks/week among women $(N=371$, postmenopausal 50-74 years) as well as $4-10$ drinks/week and 11-21 drinks/week among men $(N=370$, aged 40-80), were associated with decreased levels of cfPWV [36, 37]. Others have also reported a J-shaped relationship between alcohol consumption and arterial stiffness [38, 39]. Among Spanish men $(n=249)$ and women $(n=252)$ free of CV disease (mean age of 56 years), from the Early Vascular Aging study [38], Gonzalez-Sanchez et al. used standardized alcohol use questions and classified alcohol drinkers into 3 categories: $\leq 30 \mathrm{~g} /$ week, $>30-70 \mathrm{~g} /$ week and $>70 \mathrm{~g} /$ week consumed over the past year. cfPWV was lowest in participants reporting $\leq 30 \mathrm{~g} /$ week ( 2 drinks/week; $5.8 \pm 1.3 \mathrm{~m} / \mathrm{s}$ ), while participants consuming $>70 \mathrm{~g} /$ week ( 5 drinks/week) had the highest cfPWV $(7.5 \pm 2.3 \mathrm{~m} / \mathrm{s})$ compared to nondrinkers $(6.3 \pm 1.9 \mathrm{~m} / \mathrm{s})$, (even after adjusting for confounding factors such as age, sex, smoking history, diet systolic blood pressure and diet). Participants consuming $>70 \mathrm{~g} /$ week also had higher baPWV values compared to nondrinkers $(13.8 \pm 2.5 \mathrm{~m} / \mathrm{s}$ vs. $12.8 \pm 2.6 \mathrm{~m} / \mathrm{s})$. Similarly, in a population-based study of Japanese men $(n=3081$, mean age of 48 years) Sasaki et al. found a J-shaped relationship between alcohol consumption/day over the last month [39]. Compared to those consuming 20-39 g/day ( $\sim 2-3$ drinks/ day), non-drinkers, those consuming $<20 \mathrm{~g} /$ day, and those consuming $\geq 60 \mathrm{~g} /$ day showed significantly higher levels of baPWV. Further, the J-shaped relationship effect of alcohol on baPWV was (1) more pronounced in men $>45$ years old but not in men younger than 45 years and (2) marginally significant in women drinkers compared to non-drinkers ( $N=812$, mean age of 47 years; [39]. Interestingly, these relationships were maintained after correcting for lipids and other cardiovascular risk factors (i.e., age, BMI, blood pressure), which may have confounded or helped explain the alcohol and PWV relationship.

In the Multi-Rural Communities Cohort Study of Koreans, free of CV disease, Kim and colleagues found that any amount of alcohol was associated with an increase in baPWV in men $(n=2121$, mean age 61.3 years), but no association was found in women $(n=3418$, mean age
59.6 years) [40]. In men, all levels of alcohol consumption ( $>0-15, \geq 15-30, \geq 30-90$, and $\geq 90 \mathrm{~g} /$ day), were associated with an increase in baPWV compared to non-drinkers. These associations were linear and remained significant in men, even after adjusting for several known CV factors such as age, job, smoking status, regular exercise, waist circumference, dietary fat, protein, carbohydrate and cholesterol intake, blood pressure, lipids, and glucose [40]. In contrast, there were no differences found in baPWV among all the female drinking groups [40].

Binge drinking, the most common pattern of excessive alcohol use, may be associated with increased arterial stiffness. Charakida et al. used several standardized questions to quantify and categorize alcohol use among young adults (17 years of age, $N=1299$ ) [41]. As a potential proxy to binge drinking, increased drinking intensity ( $\geq 10$ drinks/ typical drinking day) was significantly correlated with increased arterial stiffness compared to low-intensity drinking ( $<2$ drinks on a typical drinking day; cfPWV: $5.9 \pm 0.8$ vs. $5.7 \pm 0.6 \mathrm{~m} / \mathrm{s})$, even after adjusting for confounding variables such as gender, systolic blood pressure, smoking, lipids, body weight [41]. In this same study, there was no association between the age at which participants began consuming alcohol (i.e., 13, 15, 17 years) and cfPWV measured at 17 years old [41]. More recently, our research group determined alcohol use through standardized questionnaires and the alcohol biomarker phosphatidylethanol (Peth) in healthy young adults $(N=49$, mean age 23 years) [42]. Compared to alcohol abstainers (defined as those consuming $\leq 1$ drink/ month in the last $2-3$ years), binge drinkers (defined as those who reported $\geq 4 / 5$ drinks for women/men on the same occasion or within $2 \mathrm{~h}$ over the last month at least 2 times for more than 2 years) had higher cfPWV by $0.6 \mathrm{~m} / \mathrm{s}$ [42]. We also included a group of moderate drinkers, who reported no more than 2-3 drinks per sitting for women/men, and no more than 1-2 times/week last 5 years. Interestingly, moderate drinkers also had higher cfPWV by $0.5 \mathrm{~m} / \mathrm{s}$ than alcohol abstainers [42]. Peth levels were 0 in abstainers and moderate drinkers but shown to be $>50 \mathrm{ng} / \mathrm{ml}$ in binge drinkers. These data suggest that arterial stiffness may be increased even at moderate alcohol consumption even when Peth levels are normal.

\section{Longitudinal Cohort Studies}

Compared to cross-sectional studies, longitudinal studies measure alcohol consumption and arterial stiffness at different time points, which may provide information on the attributable alcohol progression in arterial stiffness over time and related to other factors such as aging. Several population-based longitudinal cohort studies investigated the associations between alcohol consumption and arterial stiffness [43-45]. These findings support an association 
between alcohol consumption and future arterial stiffness, with the strongest associations being those with higher levels of alcohol consumption.

Nakanishi et al. found at the study baseline, different levels of alcohol consumption were associated with increased arterial stiffness during the 9-year follow-up in middle-aged healthy Japanese men $(n=1121)$ [45]. In this study, aortic (arterial) stiffness was defined as a cfPWV $\geq 8.0 \mathrm{~m} / \mathrm{s}$. Compared to non-drinkers, those who consumed $23.0-45.9 \mathrm{~g}$ ( 1.5-3 US standard drinks), 46.0-68.9 g and $\geq 69.0 \mathrm{~g}$ ( $\sim$ drinks) of ethanol per day at baseline had a 71-109\% increase in the relative risk of developing aortic stiffness. In addition, there was a significant trend for increasing amounts of alcohol consumption, suggesting a dose-response between increased arterial stiffness and alcohol consumption [45]. Many confounders were considered at baseline, such as blood pressure level, age, body mass index, and lipids; however, the potential changes in these variables, other than age, over the 9-year period, were not considered in the relative risk analysis. This study also defined alcohol use based on a single, one-time measurement and did not consider any changes in alcohol use patterns over time.

Other longitudinal studies have re-assessed alcohol consumption over the study period [43, 44]. In a study of 1185 middle-aged Japanese men [43], Matsumoto et al. measured alcohol consumption and baPWV every 3 years over a 6-year follow-up. Alcohol consumption at each time point was defined as no alcohol consumption, light-to-moderate (1-29 g of ethanol per day), and heavy ( $\geq 30 \mathrm{~g}$ of ethanol per day). Participants were categorized as "non-drinkers", "light-to-moderate drinkers", and "heavy drinkers" with the corresponding alcohol consumption reported at least twice during follow-up [43]. The authors also considered blood pressure (normal vs. elevated: $\geq 130 / 85 \mathrm{mmHg}$ ) and evaluated 'change' in baPWV over the 6-year study. In the fully adjusted analysis, the authors found that "light-to-moderate drinking" was associated with a lower 'change' in baPWV only in participants with normal blood pressure, while the "heavy drinker" status was associated with a greater 'change' in baPWV in participants with elevated blood pressure, but not in participants with normal blood pressure [43].

In the Whitehall II cohort Study of United Kingdom civil servants ( $n=3869 ; 73 \%$ male, 93\% White), O'Neill et al. defined 'long-term alcohol use groups' by using repeated alcohol consumption assessments with an interval of 4-5 years over a 25 -year period [44]. The groups included non-drinkers, stable moderate drinkers, stable heavy drinkers, unstable moderate drinkers, unstable heavy drinkers, and former drinkers. The authors found that compared to male stable moderate drinkers (1-112 $\mathrm{g}$ of ethanol per week), male stable heavy drinkers ( $>112 \mathrm{~g}$, or 8 drinks, per week), but not other groups had higher overall cfPWV in the later follow-up period. They also found that male former drinkers and non-drinkers had a greater increase in cfPWV throughout the follow-up period compared to male stable moderate drinkers. The authors postulated that these findings were attributable to the "sick quitter" phenomenon, which subjects stopped drinking due to an illness, contributing to a higher progression rate of cfPWV. No associations between alcohol consumption and arterial stiffness were identified in women [44].

\section{Synthesis of the Findings}

The association between alcohol consumption and arterial stiffness is complex and controversial from several perspectives. First of all, the studies' discrepancies may relate to both methodological and statistical issues in the assessment of alcohol consumption. Among the studies reporting no associations, Basdeki et al., Tisdel et al., and Mattace-Raso et al. lacked detailed assessment of alcohol consumption (i.e., the use of standardized alcohol use questionnaire or questions) [32, 34, 35]. Basdeki et al. also did not indicate the timeframe/duration of alcohol use assessment, which was also not always noted in other reviewed studies [32]. Secondly, the definitions of levels of alcohol consumption were different among studies, making it challenging to formulate thresholds for alcohol consumption and arterial stiffness. In addition, variations in the type of reference group used in the statistical model (i.e., non-drinkers or lightmoderate alcohol use group) differed among studies. While several studies suggest a J-shaped relationship between arterial stiffness and alcohol consumption, two studies found a dose-response relationship between alcohol use and arterial stiffness in men [40, 45]. These two studies had a wider range for each level of alcohol consumption and a higher cut-off for the highest levels of alcohol consumption compared to other studies. On the other hand, blood pressure may influence the subsequent changes in arterial stiffness, and the J-shaped relationship may only exist in individuals with normal blood pressure [43]. It may also depend on sex [39, 44]. Unfortunately, few studies included both men and women and therefore were unable to perform a separate analysis by sex. Most of the studies suggest that (1) a greater amount of alcohol consumption may be associated with arterial stiffening, as increased arterial stiffness was observed as alcohol consumption of more than 8 drinks/week (or more than 1 drink/day) [40, 43-45]; and (2) a binge pattern of alcohol use ( $4 / 5$ drinks or more for women/men on the same occasion or in one day as a proxy) may be associated with arterial stiffening [38-42, 45]. 


\section{Mechanisms of Alcohol-Associated Changes in Arterial Stiffening}

To our knowledge few studies have examined mechanisms associated with lower arterial stiffness. Concerning the alcohol-arterial stiffness and J-shaped relationship, one potential mechanism may be related to the flavonoid composition of alcoholic beverages. Others have found that total anthocyanin intake (a flavonoid) was associated with lower PWV in women (18-75 years) [46]. In our review, studies reporting a J-shaped relationship assessed alcohol consumption based upon alcoholic beverages; however, no comparisons were made among different alcoholic beverages on arterial stiffness $[39,40]$. Another potential mechanism might be related to the effects of alcohol use on lipids, such as high-density lipoprotein cholesterol (HDL-C) and the triglyceride-tohigh-density lipoprotein cholesterol (TG/HDL-c) ratio. In adults, decreased HDL levels and a higher TG/HDL-C ratio is associated with CV disease. In children (10-26 years), Urbina and colleagues found a significant association between increases in the TG/HDL-C ratio and arterial stiffness [47]. In many of the studies reviewed herein, the statistical analysis accounted for cholesterol levels, and associations remained after adjustments for serum lipid levels. One study reviewed herein found that acute alcohol consumption was associated with a decrease in arterial stiffness and a corresponding decrease in blood pressure [31], which could mechanistically explain the decrease in PWV. However, Nishiwaki et al. despite reporting a decrease in PWV following acute alcohol consumption reported no change in blood pressure [30]. The mechanisms underlying the association between regular alcohol use and lower arterial stiffness remain unknown.

The alcohol-induced arterial stiffening is attributable to structural changes of the arterial wall, such as the degradation of elastin induced by matrix metalloproteinase (MMP). In humans, blood MMP-9 levels and MMP-9 gene polymorphisms are positively associated with elastase activity and cfPWV $[48,49]$. Sillanaukee et al. found that men with alcohol consumption of $>1000$ g per week $(\sim 71$ standard drinks) had a higher level of serum MMP-9 compared to social drinkers $(<200 \mathrm{~g}$ per week) [50]. The increases in serum MMP-9 levels can increase the degradation of elastin, cause structural changes of the arterial wall, and thereby increase arterial stiffness. Using a rat model, Koken et al. also found that chronic heavy alcohol consumption ( $1 \mathrm{ml}$ of $80 \%$ ethanol (v/v) in distilled water by gavage for 30 days) increased blood MMP-9 levels. The increases in MMP-9 levels were restored by intraperitoneal Vitamin E administration [51], suggesting an oxidative stress mechanism of alcohol-induced arterial stiffening.
Indeed, oxidative stress, the excessive production of reactive oxygen species relative to antioxidant defenses, plays a key role in alcohol-induced vascular damages and dysfunctions [52]. Animal studies suggest that one-time and repeated heavy alcohol consumption is associated with the excessive production of reactive oxygen species. As evidenced by increased plasma levels of thiobarbituric acidreacting substance (a marker of systemic lipoperoxidation), superoxide anion levels, and membrane-to-cytosol fraction expression of the $\mathrm{p} 47$ phox subunit of nicotinamide adenine dinucleotide phosphate oxidase in vascular beds [53-55]. These reactive oxygen species directly or indirectly decrease nitric oxide, a vasodilator derived from vascular endothelial cells. Decreased bioavailability of nitric oxide, or endothelial dysfunction causes decreased vasodilation and increased vasoconstriction (or vascular smooth muscle cell tone), causing arterial stiffening. In normotensive men, nitric oxide synthase inhibitor infusion decreases nitric oxide production and acutely increases cfPWV [56]. Therefore, alcoholinduced arterial stiffening may be related to endothelial dysfunction. Along with this possibility, our recent systemic review suggests that heavy alcohol consumption $(\geq 3-5$ drinks, daily or binge pattern) is associated with a decrease in brachial artery flow-mediated dilation [57], a non-invasive measure of endothelial function in humans.

Increases in vascular smooth muscle cell tone or stiffness can also result from the activation of sympathetic nerve activity. Experimental studies demonstrate that a single excessive bout of alcohol use, or binge-like drinking episode, transiently enhanced sympathetic nerve activity, directly measured by microneurography [58-65]. Our recent cross-sectional study also demonstrated that repeated binge drinking was associated with increased 24-h urinary norepinephrine levels, and higher norepinephrine levels were correlated to higher cfPWV values [42]. Norepinephrine is a marker of sympathetic activity [66] and increases vascular smooth muscle tone leading to vasoconstriction [67]. In normotensive male adults, norepinephrine infusion acutely increases cfPWV [56]. Norepinephrine can also induce arterial stiffening via vascular remodeling, thickening the vascular wall by increasing the number of vascular smooth muscle cells (hyperplasia), and deposition of both collagen and elastin. [68]. All these factors lead to of high blood pressure and further arterial stiffening. While heavy alcohol consumption is associated with an increased risk of hypertension [69], other factors, including arterial calcification [70] and inflammation [71], can also contribute to alcohol-induced arterial stiffening (Fig. 2) [72]. 


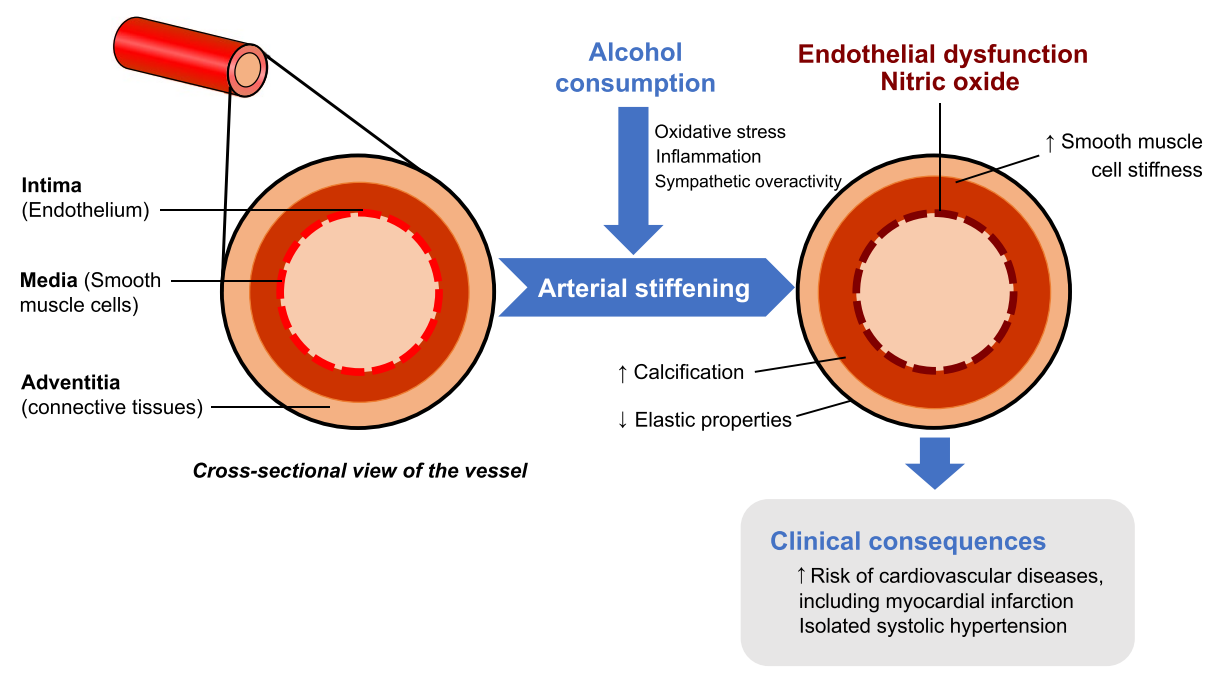

Fig. 2 Mechanisms of alcohol-induced arterial stiffening. Alcoholinduced arterial stiffness is mediated by activation of oxidative stress and inflammatory factors as well as sympathetic overactivity; all of which are attributable to a decrease in nitric oxide and endothelial dysfunction. Endothelial dysfunction is characterized by inadequate or abnormal microvascular vasodilator or constrictor response to physiologic stimuli (e.g., flow), pathophysiologic stimuli (e.g., high pressure) and circulating stimuli (e.g., neurohormones). Increased

\section{Conclusions}

Collectively, data from many studies reviewed herein suggest a relationship between alcohol consumption and arterial stiffness. In particular, higher levels of alcohol consumption or a binge pattern of consumption is associated with arterial stiffening. Most of the published studies were cross-sectional, did not consider other factors that may also influence arterial stiffness over time (salt intake and physical activity), lacked detailed assessment of alcohol consumption and PWV. These methodological inconsistencies make it difficult to generate specific conclusions. Finally, sex and/or race may influence the relationship between alcohol consumption and arterial stiffness and requires further investigation. Given the significant value of arterial stiffness in the predicting $\mathrm{CV}$ disease, it is important to include alcohol consumption assessment into routine clinical practice to manage $\mathrm{CV}$ disease.

Author Contributions Mariann R. Piano, Chueh-Lung Hwang, and Shane A. Phillips had the idea for the article topic. Mariann R. Piano, Chueh-Lung Hwang, James Muchira, and Shane A. Phillips performed the literature search. Chueh-Lung Hwang, James Muchira, Brooks A. Hibner, Shane A. Phillips and Mariann R. Piano all participated in the critique and analysis of articles. Chueh-Lung Hwang, James Muchira, Brooks Hibner, and Mariann R. Piano participated in the draft and/or critically revised the work. sympathetic activity and increased norepinephrine levels can lead to hypertrophy and hyperplasia of smooth muscle cells leading to an increase thickness of the medial layer of the blood vessel wall. Oxidative stress also promotes inflammation via immune activation and migration of activated immune cells into the vascular wall ultimately stimulating wall remodeling which includes structural elastin abnormalities, and accumulation of stiffer collagen fibers in the arterial wall and endothelial dysfunction. Adapted from: [72]

Funding This work was supported by the NIAAA K99 AA028537 (CLH).

Data Availability Data sharing not applicable to this article as no datasets were generated or analyzed during the current study.

\section{Declarations}

Conflict of interest The authors have no competing interests to declare that are relevant to the content of this article.

\section{References}

1. Townsend, R. R., Wilkinson, I. B., Schiffrin, E. L., Avolio, A. P., Chirinos, J. A., Cockcroft, J. R., Heffernan, K. S., Lakatta, E. G., McEniery, C. M., Mitchell, G. F., Najjar, S. S., Nichols, W. W., Urbina, E. M., \& Weber, T. (2015). Recommendations for improving and standardizing vascular research on arterial stiffness: A scientific statement from the American heart association. Hypertension, 66(3), 698-722. https://doi.org/10.1161/hyp.00000 00000000033

2. Boutouyrie, P., Chowienczyk, P., Humphrey, J. D., \& Mitchell, G. F. (2021). Arterial stiffness and cardiovascular risk in hypertension. Circulation Research, 128(7), 864-886. https://doi.org/10. 1161/circresaha.121.318061

3. Mackenzie, I. S., Wilkinson, I. B., \& Cockcroft, J. R. (2002). Assessment of arterial stiffness in clinical practice. QJM, 95(2), 67-74. https://doi.org/10.1093/qjmed/95.2.67

4. Piano, M. R. (2017). Alcohol's effects on the cardiovascular system. Alcohol Research, 38(2), 219-241. 
5. Substance Abuse and Mental Health Services Administration. (2020). Key Substance Use and Mental Health Indicators in the United States: Results from the 2019 National Survey on Drug Use and Health (Hhs Publication No. Pep20-07-01-001, Nsduh Series H-55). Center for Behavioral Health Statistics and Quality, Substance Abuse and Mental Health Services Administration.

6. Barbosa, C., Cowell, A. J., \& Dowd, W. N. (2021). Alcohol consumption in response to the Covid-19 pandemic in the United States. Journal of Addiction Medicine, 15(4), 341-344. https:// doi.org/10.1097/ADM.0000000000000767

7. Pollard, M. S., Tucker, J. S., \& Green, H. D., Jr. (2020). Changes in adult alcohol use and consequences during the Covid-19 pandemic in the us. JAMA Network Open, 3(9), e2022942. https://doi. org/10.1001/jamanetworkopen.2020.22942

8. Nichols, W. W., O'Rourke, M., \& Vlachopoulos, C. (2011). Mcdonald's Blood Flow in Arteries: Theoretical, Experimental and Clinical Principles (6th ed.). CRC Press.

9. Smith, D. L., \& Fernhall, B. (2011). Advanced Cardiovascular Exercise Physiology. Human Kinetics.

10. Sherratt, M. J. (2009). Tissue elasticity and the ageing elastic fibre. Age, 31(4), 305-325. https://doi.org/10.1007/s11357-009-9103-6

11. Arribas, S. M., Hinek, A., \& González, M. C. (2006). Elastic fibres and vascular structure in hypertension. Pharmacology \& Therapeutics, 111(3), 771-791. https://doi.org/10.1016/j.pharm thera.2005.12.003

12. Segers, P., Rietzschel, E. R., \& Chirinos, J. A. (2020). How to measure arterial stiffness in humans. Arteriosclerosis, Thrombosis, and Vascular Biology, 40(5), 1034-1043. https://doi.org/10. 1161/ATVBAHA.119.313132

13. The Reference Values for Arterial Stiffness, C. (2010). Determinants of pulse wave velocity in healthy people and in the presence of cardiovascular risk factors: 'Establishing normal and reference values.' European Heart Journal, 31(19), 2338-2350. https://doi. org/10.1093/eurheartj/ehq165

14. Tanaka, H. (2017). Various indices of arterial stiffness: are they closely related or distinctly different? Pulse, 5(1-4), 1-6. https:// doi.org/10.1159/000461594

15. Guerin, A. P., Blacher, J., Pannier, B., Marchais, S. J., Safar, M. E., \& London, G. M. (2001). Impact of aortic stiffness attenuation on survival of patients in end-stage renal failure. Circulation, 103(7), 987-992. https://doi.org/10.1161/01.CIR.103.7.987

16. Choi, C. U., Park, E. B., Suh, S. Y., Kim, J. W., Kim, E. J., Rha, S. W., Seo, H. S., Oh, D. J., \& Park, C. G. (2007). Impact of aortic stiffness on cardiovascular disease in patients with chest pain: Assessment with direct intra-arterial measurement. American Journal of Hypertension, 20(11), 1163-1169. https://doi.org/10. 1016/j.amjhyper.2007.07.004

17. Cruickshank, K., Riste, L., Anderson, S. G., Wright, J. S., Dunn, G., \& Gosling, R. G. (2002). Aortic pulse-wave velocity and its relationship to mortality in diabetes and glucose intolerance: An integrated index of vascular function? Circulation, 106(16), 2085-2090. https://doi.org/10.1161/01.CIR.0000033824.02722. F7

18. Ben-Shlomo, Y., Spears, M., Hwang, S.-J., Lakatta, E. G., Laurent, S., Maldonado, J., Mitchell, G. F., Najjar, S. S., Newman, A. B., Ohishi, M., Pannier, B., Pereira, T., Boustred, C., Vasan, R. S., Shokawa, T., Sutton-Tyrell, K., Verbeke, F., Wang, K.-L., Webb, D. J., ... \& Cruickshank, J. K. (2014). Aortic pulse wave velocity improves cardiovascular event prediction: An individual participant meta-analysis of prospective observational data from 17,635 subjects. Journal of the American College of Cardiology, 63(7), 636-646. https://doi.org/10.1016/j.jacc.2013.09.063

19. Laurent, S., Boutouyrie, P., Asmar, R., Gautier, I., Laloux, B., Guize, L., Ducimetiere, P., \& Benetos, A. (2001). Aortic stiffness is an independent predictor of all-cause and cardiovascular mortality in hypertensive patients. Hypertension, 37(5), 12361241. https://doi.org/10.1161/01.hyp.37.5.1236

20. Laurent, S., Katsahian, S., Fassot, C., Tropeano, A. I., Gautier, I., Laloux, B., \& Boutouyrie, P. (2003). Aortic stiffness is an independent predictor of fatal stroke in essential hypertension. Stroke, 34(5), 1203-1206. https://doi.org/10.1161/01.Str.00000 65428.03209 .64

21. Boutouyrie, P., Tropeano, A. I., Asmar, R., Gautier, I., Benetos, A., Lacolley, P., \& Laurent, S. (2002). Aortic stiffness is an independent predictor of primary coronary events in hypertensive patients: a longitudinal study. Hypertension, 39(1), 10-15. https:// doi.org/10.1161/hy0102.099031

22. Meaume, S., Rudnichi, A., Lynch, A., Bussy, C., Sebban, C., Benetos, A., \& Safar, M. E. (2001). Aortic pulse wave velocity as a marker of cardiovascular disease in subjects over 70 years old. Journal of Hypertension, 19(5), 871-877. https://doi.org/10.1097/ 00004872-200105000-00006

23. Mattace-Raso, F. U. S., Van Der Cammen, T. J. M., Witteman, J. C. M., Hofman, A., Van Popele, N. M., Bos, M. L., Schalekamp, M. A. D. H., Asmar, R., Reneman, R. S., Hoeks, A. P. G., \& Breteler, M. M. B. (2006). Arterial stiffness and risk of coronary heart disease and stroke : the Rotterdam study. Circulation, 113(5), 657-663. https://doi.org/10.1161/CIRCULATIONAHA. 105.555235

24. Willum Hansen, T., Staessen, J. A., Torp-Pedersen, C., Rasmussen, S., Thijs, L., Ibsen, H., \& Jeppesen, J. (2006). Prognostic value of aortic pulse wave velocity as index of arterial stiffness in the general population. Circulation, 113(5), 664-670. https://doi. org/10.1161/CIRCULATIONAHA.105.579342

25. Van Bortel, L. M., Laurent, S., Boutouyrie, P., Chowienczyk, P., Cruickshank, J. K., De Backer, T., Filipovsky, J., Huybrechts, S., Mattace-Raso, F. U., Protogerou, A. D., Schillaci, G., Segers, P., Vermeersch, S., Weber, T., Artery, S., European Society of Hypertension Working Group on Vascular, S., Function, \& European Network for Noninvasive Investigation of Large, A. (2012). Expert consensus document on the measurement of aortic stiffness in daily practice using carotid-femoral pulse wave velocity. Journal of Hypertension, 30(3), 445-448. https://doi.org/10.1097/HJH. 0b013e $32834 \mathrm{fa} 8 \mathrm{~b} 0$

26. Reference Values for Arterial Stiffness, C. (2010). Determinants of pulse wave velocity in healthy people and in the presence of cardiovascular risk factors: "Establishing normal and reference values." European Heart Journal, 31(19), 2338-2350. https://doi. org/10.1093/eurheartj/ehq165

27. McEniery, C. M., Yasmin, N., Hall, I. R., Qasem, A., Wilkinson, I. B., Cockcroft, J. R., Investigators, A. (2005). Normal vascular aging: differential effects on wave reflection and aortic pulse wave velocity: The anglo-cardiff collaborative trial (Acct). Journal of the American College of Cardiology, 46(9), 1753-1760. https:// doi.org/10.1016/j.jacc.2005.07.037

28. Tanaka, H., Munakata, M., Kawano, Y., Ohishi, M., Shoji, T., Sugawara, J., Tomiyama, H., Yamashina, A., Yasuda, H., Sawayama, T., \& Ozawa, T. (2009). Comparison between carotid-femoral and brachial-ankle pulse wave velocity as measures of arterial stiffness. Journal of Hypertension, 27(10), 2022-2027. https://doi. org/10.1097/HJH.0b013e32832e94e7

29. Laurent, S., Cockcroft, J., Van Bortel, L., Boutouyrie, P., Giannattasio, C., Hayoz, D., Pannier, B., Vlachopoulos, C., Wilkinson, I., $\&$ Struijker-Boudier, H. (2006). Expert consensus document on arterial stiffness: Methodological issues and clinical applications. European Heart Journal, 27(21), 2588-2605. https://doi.org/10. 1093/eurheartj/ehl254

30. Nishiwaki, M., Kora, N., \& Matsumoto, N. (2017). Ingesting a small amount of beer reduces arterial stiffness in healthy humans. Physiological Reports, 5(15), 13381. https://doi.org/10.14814/ phy 2.13381 
31. Mahmud, A., \& Feely, J. (2002). Divergent effect of acute and chronic alcohol on arterial stiffness. American Journal of Hypertension, 15(3), 240-243. https://doi.org/10.1016/s0895-7061(01) 02315-9

32. Basdeki, E. D., Tsirimiagkou, C., Argyris, A., Moschonis, G., Sfikakis, P., Protogerou, A. D., \& Karatzi, K. (2021). Moderately increased alcohol consumption is associated with higher pressure wave reflections and blood pressure in men. Nutrition, Metabolism, and Cardiovascular Diseases, 31(1), 85-94. https://doi.org/ 10.1016/j.numecd.2020.08.013

33. Yu, A., Cooke, A. B., Scheffler, P., Doonan, R. J., \& Daskalopoulou, S. S. (2021). Alcohol exerts a shifted u-shaped effect on central blood pressure in young adults. Journal of General Internal Medicine, 36(10), 2975-2981. https://doi.org/10.1007/ s11606-021-06665-0

34. Tisdel, D. M., Gadberry, J. J., Burke, S. L., Carlini, N. A., Fleenor, B. S., \& Campbell, M. S. (2021). Dietary fat and alcohol in the prediction of indices of vascular health among young adults. Nutrition, 84, 111120. https://doi.org/10.1016/j.nut.2020.111120

35. Mattace-Raso, F. U., van der Cammen, T. J., van den Elzen, A. P., Schalekamp, M. A., Asmar, R., Reneman, R. S., Hoeks, A. P., Hofman, A., \& Witteman, J. C. (2005). Moderate alcohol consumption is associated with reduced arterial stiffness in older adults: The Rotterdam study. Journal of Gerontology Series A, 60(11), 1479-1483. https://doi.org/10.1093/gerona/60.11.1479

36. Sierksma, A., Lebrun, C. E., van der Schouw, Y. T., Grobbee, D. E., Lamberts, S. W., Hendriks, H. F., \& Bots, M. L. (2004). Alcohol consumption in relation to aortic stiffness and aortic wave reflections: A cross-sectional study in healthy postmenopausal women. Arteriosclerosis, Thrombosis, and Vascular Biology, 24(2), 342-348. https://doi.org/10.1161/01.ATV.0000110784. $52412.8 \mathrm{f}$

37. Sierksma, A., Muller, M., van der Schouw, Y. T., Grobbee, D. E., Hendriks, H. F., \& Bots, M. L. (2004). Alcohol consumption and arterial stiffness in men. Journal of Hypertension, 22(2), 357-362. https://doi.org/10.1097/00004872-200402000-00020

38. Gonzalez-Sanchez, J., Garcia-Ortiz, L., Rodriguez-Sanchez, E., Maderuelo-Fernandez, J. A., Tamayo-Morales, O., LugonesSanchez, C., Recio-Rodriguez, J. I., \& Gomez-Marcos, M. A. (2020). The relationship between alcohol consumption with vascular structure and arterial stiffness in the Spanish population: Eva study. Alcoholism, Clinical and Experimental Research, 44(9), 1816-1824. https://doi.org/10.1111/acer.14411

39. Sasaki, S., Yoshioka, E., Saijo, Y., Kita, T., Okada, E., Tamakoshi, A., \& Kishi, R. (2013). Relation between alcohol consumption and arterial stiffness: A cross-sectional study of middle-aged Japanese women and men. Alcohol, 47(8), 643-649. https://doi. org/10.1016/j.alcohol.2013.10.003

40. Kim, M. K., Shin, J., Kweon, S. S., Shin, D. H., Lee, Y. H., Chun, B. Y., \& Choi, B. Y. (2014). Harmful and beneficial relationships between alcohol consumption and subclinical atherosclerosis. Nutrition, Metabolism, and Cardiovascular Diseases, 24(7), 767-776. https://doi.org/10.1016/j.numecd.2014.02.004

41. Charakida, M., Georgiopoulos, G., Dangardt, F., Chiesa, S. T., Hughes, A. D., Rapala, A., Davey Smith, G., Lawlor, D., Finer, N., \& Deanfield, J. E. (2019). Early vascular damage from smoking and alcohol in teenage years: the Alspac study. European Heart Journal, 40(4), 345-353. https://doi.org/10.1093/eurheartj/ehy524

42. Hwang, C. L., Piano, M. R., Thur, L. A., Peters, T. A., da Silva, A. L. G., \& Phillips, S. A. (2020). The effects of repeated binge drinking on arterial stiffness and urinary norepinephrine levels in young adults. Journal of Hypertension, 38(1), 111-117. https:// doi.org/10.1097/hjh.0000000000002223

43. Matsumoto, C., Tomiyama, H., Yamada, J., Yoshida, M., Shiina, K., Nagata, M., \& Yamashina, A. (2009). Association of blood pressure levels with the effects of alcohol intake on the vasculature in Japanese men. Hypertension Research, 32(2), 127-132. https:// doi.org/10.1038/hr.2008.23

44. O’Neill, D., Britton, A., Brunner, E. J., \& Bell, S. (2017). Twentyfive-year alcohol consumption trajectories and their association with arterial aging: A prospective cohort study. Journal of the American Heart Association, 6(2), e005288. https://doi.org/10. 1161/jaha.116.005288

45. Nakanishi, N., Kawashimo, H., Nakamura, K., Suzuki, K., Yoshida, H., Uzura, S., \& Tatara, K. (2001). Association of alcohol consumption with increase in aortic stiffness: A 9-year longitudinal study in middle-aged Japanese men. Industrial Health, 39(1), 24-28. https://doi.org/10.2486/indhealth.39.24

46. Jennings, A., Welch, A. A., Fairweather-Tait, S. J., Kay, C., Minihane, A. M., Chowienczyk, P., Jiang, B., Cecelja, M., Spector, T., Macgregor, A., \& Cassidy, A. (2012). Higher anthocyanin intake is associated with lower arterial stiffness and central blood pressure in women. American Journal of Clinical Nutrition, 96(4), 781-788. https://doi.org/10.3945/ajcn.112.042036

47. Urbina, E. M., Khoury, P. R., McCoy, C. E., Dolan, L. M., Daniels, S. R., \& Kimball, T. R. (2013). Triglyceride to Hdl-C ratio and increased arterial stiffness in children, adolescents, and young adults. Pediatrics, 131(4), e1082-1090. https://doi.org/10.1542/ peds.2012-1726

48. Yasmin, W., McEniery, C. M., Wallace, S., Dakham, Z., Pulsalkar, P., Maki-Petaja, K., Ashby, M. J., Cockcroft, J. R., \& Wilkinson, I. B. (2005). Matrix metalloproteinase-9 (Mmp-9), Mmp-2, and serum elastase activity are associated with systolic hypertension and arterial stiffness. Arteriosclerosis, Thrombosis, and Vascular Biology, 25(2), 372. https://doi.org/10.1161/01.ATV.0000151373. 33830.41

49. Yasmin, W., McEniery, C. M., O'Shaughnessy, K. M., Harnett, P., Arshad, A., Wallace, S., Maki-Petaja, K., McDonnell, B., Ashby, M. J., Brown, J., Cockcroft, J. R., \& Wilkinson, I. B. (2006). Variation in the human matrix metalloproteinase-9 gene is associated with arterial stiffness in healthy individuals. Arteriosclerosis, Thrombosis, and Vascular Biology, 26(8), 1799-1805. https://doi. org/10.1161/01.ATV.0000227717.46157.32

50. Sillanaukee, P., Kalela, A., Seppa, K., Hoyhtya, M., \& Nikkari, S. T. (2002). Matrix metalloproteinase-9 is elevated in serum of alcohol abusers. The European Journal of Clinical Investigation, 32(4), 225-229. https://doi.org/10.1046/j.1365-2362.2002. 00975.x

51. Koken, T., Gursoy, F., \& Kahraman, A. (2010). Long-term alcohol consumption increases pro-matrix metalloproteinase-9 levels via oxidative stress. Journal of Medical Toxicology, 6(2), 126-130. https://doi.org/10.1007/s13181-010-0081-y

52. Phillips, S. A., Osborn, K., Hwang, C. L., Sabbahi, A., \& Piano, M. R. (2019). Ethanol induced oxidative stress in the vasculature: Friend or foe. Current Hypertension Reviews, 16(3), 181-191. https://doi.org/10.2174/1573402115666190325124622

53. Simplicio, J. A., Hipolito, U. V., Vale, G. T., Callera, G. E., Pereira, C. A., Touyz, R. M., Tostes, R. C., \& Tirapelli, C. R. (2016). Acute ethanol intake induces $\mathrm{Nad}(\mathrm{P}) \mathrm{H}$ oxidase activation and rhoa translocation in resistance arteries. Arquivos Brasileiros de Cardiologia, 107(5), 427-436. https://doi.org/10.5935/abc. 20160147

54. Yogi, A., Callera, G. E., Mecawi, A. S., Batalhao, M. E., Carnio, E. C., Antunes-Rodrigues, J., Queiroz, R. H., Touyz, R. M., \& Tirapelli, C. R. (2012). Acute Ethanol intake induces superoxide anion generation and mitogen-activated protein kinase phosphorylation in rat aorta: A role for angiotensin type 1 receptor. Toxicology and Applied Pharmacology, 264(3), 470-478. https://doi. org/10.1016/j.taap.2012.08.029

55. Husain, K., Vazquez, M., Ansari, R. A., Malafa, M. P., \& Lalla, J. (2008). Chronic alcohol-induced oxidative endothelial injury relates to angiotensin II levels in the rat. Molecular and 
Cellular Biochemistry, 307(1-2), 51-58. https://doi.org/10.1007/ s11010-007-9583-6

56. Stewart, A. D., Millasseau, S. C., Kearney, M. T., Ritter, J. M., \& Chowienczyk, P. J. (2003). Effects of inhibition of basal nitric oxide synthesis on carotid-femoral pulse wave velocity and augmentation index in humans. Hypertension, 42(5), 915-918. https://doi.org/10.1161/01.HYP.0000092882.65699.19

57. Hwang, C. L., Piano, M. R., \& Phillips, S. A. (2021). The effects of alcohol consumption on flow-mediated dilation in humans: a systematic review. Physiological Reports, 9(10), 14872. https:// doi.org/10.14814/phy2.14872

58. Spaak, J., Merlocco, A. C., Soleas, G. J., Tomlinson, G., Morris, B. L., Picton, P., Notarius, C. F., Chan, C. T., \& Floras, J. S. (2008). Dose-related effects of red wine and alcohol on hemodynamics, sympathetic nerve activity, and arterial diameter. American Journal of Physiology-Heart and Circulatory Physiology, 294(2), H605-H612. https://doi.org/10.1152/ajpheart.01162.2007

59. Grassi, G. M., Somers, V. K., Renk, W. S., Abboud, F. M., \& Mark, A. L. (1989). Effects of alcohol intake on blood pressure and sympathetic nerve activity in normotensive humans: A preliminary report. Journal of Hypertension, 7, S20-21. https://doi. org/10.1097/00004872-198900076-00007

60. Carter, J. R., Stream, S. F., Durocher, J. J., \& Larson, R. A. (2011). Influence of acute alcohol ingestion on sympathetic neural responses to orthostatic stress in humans. American Journal of Physiology, 300(5), E771-778. https://doi.org/10.1152/ajpendo. 00674.2010

61. Andrade, A. C., Cesena, F. H., Consolim-Colombo, F. M., Coimbra, S. R., Benjo, A. M., Krieger, E. M., \& Luz, P. L. (2009). Short-term red wine consumption promotes differential effects on plasma levels of high-density lipoprotein cholesterol, sympathetic activity, and endothelial function in hypercholesterolemic, hypertensive, and healthy subjects. Clinics, 64(5), 435-442. https://doi. org/10.1590/s1807-59322009000500011

62. Hering, D., Kucharska, W., Kara, T., Somers, V. K., \& Narkiewicz, K. (2011). Potentiated sympathetic and hemodynamic responses to alcohol in hypertensive vs. normotensive individuals. Journal of Hypertension, 29(3), 537-541. https://doi.org/10.1097/HJH. $0 \mathrm{~b} 013 \mathrm{e} 328342 \mathrm{~b} 2 \mathrm{a} 9$

63. Iwase, S., Matsukawa, T., Ishihara, S., Tanaka, A., Tanabe, K., Danbara, A., Matsuo, M., Sugiyama, Y., \& Mano, T. (1995). Effect of oral ethanol intake on muscle sympathetic nerve activity and cardiovascular functions in humans. Journal of the Autonomic Nervous System, 54(3), 206-214.

64. Randin, D., Vollenweider, P., Tappy, L., Jequier, E., Nicod, P., \& Scherrer, U. (1995). Suppression of alcohol-induced hypertension by dexamethasone. New England Journal of Medicine, 332(26), 1733-1737. https://doi.org/10.1056/NEJM199506293322601

65. van de Borne, P., Mark, A. L., Montano, N., Mion, D., \& Somers, V. K. (1997). Effects of alcohol on sympathetic activity, hemodynamics, and chemoreflex sensitivity. Hypertension, 29(6), $1278-1283$.

66. Joyner, M. J. (2016). Preclinical and clinical evaluation of autonomic function in humans. Journal of Physiology, 594(14), 40094013. https://doi.org/10.1113/JP271875

67. Girerd, X., Chamiot-Clerc, P., Copie, X., Renaud, J. F., Laurent, S., \& Safar, M. E. (1998). Effects of norepinephrine on the mechanical properties of the human radial artery in vitro. American Heart Journal, 136(4), 624-631.

68. Dao, H. H., Lemay, J., de Champlain, J., deBlois, D., \& Moreau, P. (2001). Norepinephrine-induced aortic hyperplasia and extracellular matrix deposition are endothelin-dependent. Journal of Hypertension, 19(11), 1965-1973. https://doi.org/10.1097/00004 872-200111000-00006

69. Piano, M. R., Thur, L. A., Hwang, C. L., \& Phillips, S. A. (2020). Effects of alcohol on the cardiovascular system in women. Alcohol Research, 40(2), 12. https://doi.org/10.35946/arcr.v40.2.12

70. Pletcher, M. J., Varosy, P., Kiefe, C. I., Lewis, C. E., Sidney, S., \& Hulley, S. B. (2005). Alcohol consumption, binge drinking, and early coronary calcification: findings from the coronary artery risk development in young adults (cardia) study. American Journal of Epidemiology, 161(5), 423-433. https://doi.org/10.1093/aje/ kwi062

71. Gonzalez-Reimers, E., Santolaria-Fernandez, F., Martin-Gonzalez, M. C., Fernandez-Rodriguez, C. M., \& Quintero-Platt, G. (2014). Alcoholism: A systemic proinflammatory condition. World Journal of Gastroenterology, 20(40), 14660-14671. https:// doi.org/10.3748/wjg.v20.i40.14660

72. Costantino, S., Paneni, F., \& Cosentino, F. (2016). Ageing, metabolism and cardiovascular disease. Journal of Physiology, 594(8), 2061-2073. https://doi.org/10.1113/jp270538

73. Jeroncic, A., Gunjaca, G., Mrsic, D. B., Mudnic, I., Brizic, I., Polasek, O., \& Boban, M. (2016). Normative equations for central augmentation index: assessment of inter-population applicability and how it could be improved. Scientific Reports, 6, 27016. https:// doi.org/10.1038/srep27016

Publisher's Note Springer Nature remains neutral with regard to jurisdictional claims in published maps and institutional affiliations. 